\title{
Intervención, innovación e investigación. Una relación necesaria para las didácticas*
}

\section{Anna Camps**}

\section{Resumen}

El artículo plantea la interrelación entre las prácticas de aula (intervención), la innovación y la investigación. Se argumenta la necesidad de la investigación en didáctica de la lengua inserta en el sistema didáctico, que es dinámico y que, por lo tanto, cambia en el tiempo y depende del contexto. Se plantea, pues, la innovación como adaptación a los nuevos contextos que se generan por los cambios en los sistemas implicados. Los procesos dinámicos de enseñanza y aprendizaje pueden ser objeto de análisis e investigación a distintos niveles: desde la observación de disfunciones que llevan a cambios en la actuación, hasta el análisis sistemático que dará lugar a la elaboración de nuevo conocimiento que podrá ser compartido por la comunidad docente.

Palabras clave: didáctica de la lengua, sistema didáctico, intervención educativa, innovación, niveles de investigación en didáctica de la lengua.

\section{Abstract}

This paper looks at the relationship between classroom practices (intervention), innovation and research. It argues the need for research in language teaching inserted in the training system, which is dynamic and, therefore, changes over time and depends on the context. There is innovation and adaptation to new contexts that are generated by changes in the systems involved. The dynamic processes of teaching and learning can be analysed and researched at various levels from the observation of dysfunctions that lead to changes in performance to the level of systematic analysis that will lead to develop new knowledge that can be shared by the educational community.

Keywords: language teaching, educational system, educational intervention, innovation, research levels in language teaching.

Los tres temas que configuran el título representan conceptos poco definidos o, como mínimo, de una cierta ambigüedad.

Comenzaré por definir el primero, para aclarar el lugar que ocupa la intervención y de qué manera la veo ligada a la innovación, para finalizar hablando de la complejidad de la investigación en didáctica de la lengua. Por supuesto que, a pesar de que ordeno las ideas básicas siguiendo este eje, la reflexión es conjunta.

* Traducido con permiso de la autora por María Santamarina. La versión original de este trabajo se presentó en catalán en el II Congreso Internacional de Didácticas celebrado en Girona (España) en 2010.

** Anna Camps es Licenciada en Filosofía y letras (filología catalana) y en Pedagogía por la UB. Doctora en Psicología por la UB.En la actualidad, es catedrática emérita de la Universitat Autònoma de Barcelona.(UAB) 
Partiré de la base de que la didáctica de la lengua tiene como objeto la relación compleja que se da entre el docente, el alumno y la lengua o las lenguas que se enseñan, se aprenden y se usan para enseñar y aprender, es lo que se conoce como sistema didáctico. El objetivo que persigue es elaborar conocimiento sobre este objeto para mejorar el de los aprendices sobre las lenguas, que es la finalidad de su enseñanza en la escuela.

Desde este enfoque, es evidente que el conocimiento que se elabora en el campo de la didáctica de la lengua está y ha de estar orientado a la práctica, es decir, a la intervención. La enseñanza y el aprendizaje de las lenguas son el origen y la finalidad de la investigación en este campo. Esta finalidad se ha entendido a menudo como aplicación de conocimientos teóricos elaborados en otros campos, en el supuesto de que la práctica no podía ser objeto de conocimiento específico. Un planteamiento como este dejaría cualquier actividad humana fuera de las posibilidades del conocimiento sistemático.

\section{Las actividades humanas como objeto de investigación}

Si consideramos la enseñanza y el aprendizaje de las lenguas como actividades humanas compartidas, las cosas cambian y se vuelven más complejas. Concebimos la actividad humana como un sistema a través del cual las personas actúan sobre los objetos con el fin de obtener algún resultado. Siguiendo a Leontiev (1972 [1981]) "la actividad es un formato social organizador y regulador del comportamiento humano" y se aplica a cualquier organización colectiva de los comportamientos, orientada hacia una finalidad, en la cual se inscriben las interacciones de los individuos con el medio. Las actividades se distinguen según la finalidad en torno a la cual se articulan y tienen un motivo, lo que estimula la acción, que tiene su origen en las condiciones sociales en que se desarrolla la actividad.
Siguiendo a Engeström (1987), podemos decir que el sistema didáctico es un sistema de sistemas de actividad que no es estable, más bien al contrario, este sistema de sistemas en el que interactúan los polos de la enseñanza, del aprendizaje y de los contenidos que se enseñan y se aprenden es fundamentalmente dinámico. La imagen del triángulo que Engeström utiliza para representarlo no traduce esta característica fundamental con suficiente énfasis: los sistemas implicados tienen un motivo (aquello que mueve a actuar) y tienden a una finalidad. Esto implica desarrollo en el tiempo. El tiempo didáctico es un tema muy tratado, especialmente en el ámbito francófono. Sin embargo quizás no se ha insistido lo suficiente en la interrelación de las actividades en el proceso dinámico implica la interrelación de sistemas en el sistema didáctico.

Pongamos un ejemplo: en las situaciones de aprendizaje de los géneros escritos, cuando se propone a los alumnos que escriban textos que tengan un objetivo comunicativo (escribir un artículo en una revista, una carta, etc.), estos textos, además de esta función, tienen otra que es la propia de la escuela: el aprendizaje de unos contenidos específicos referidos al género discursivo que se trabaja. Así pues, los alumnos se ven inmersos en dos sistemas de actividades con objetivos diferentes, a veces contrapuestos, pero siempre interrelacionados: comunicar y aprender (cf. Ribas, 2001; Camps y Uribe, 2008). En el marco de la actividad del aprendizaje, se dan, o se pueden dar, situaciones comunicativas específicas que, muchas veces, se refieren a la actividad discursiva consistente en la producción textual, lo cual da lugar a usos metadiscursivos del lenguaje.

Los sistemas que interactúan en el sistema didáctico son cambiantes. El ejemplo anterior nos lo sugiere; por ejemplo, las situaciones comunicativas que pueden dar lugar a situaciones de escritura en la escuela son socialmente cambiantes (niveles de formalidad diferentes según el medio, formas nuevas, géneros 
nuevos, etc.). Podemos ampliar los ejemplos de sistemas implicados que cambian y que evolucionan: los contenidos de la enseñanza de lenguas se ven influidos por la evolución y el cambio en los estudios lingüísticos; las lenguas que la escuela tiene que tomar en consideración se diversifican (por la globalización, por las lenguas que vienen de otros países, etc.); las condiciones sociales y familiares del alumno son cambiantes (situaciones socioeconómicas, familiares, de los entornos urbanos, etc.); los instrumentos mediadores experimentan transformaciones importantes: libros de texto, herramientas informáticas, etc. Por otro lado, la reflexión, la posible investigación sobre el mismo sistema puede conllevar la necesidad de introducir cambios que se hacen cuando se detecta que algo no funciona, que no se ajusta a los objetivos que la enseñanza se plantea. $\mathrm{Si}$ consideramos que los sistemas son dinámicos, necesariamente habrá que tener en cuenta que se tienen que adaptar a las condiciones cambiantes en que se desarrollan las actividades.

Con todo esto quiero destacar que la actividad de enseñar, insertada en el sistema de actividad tal como lo hemos presentado, no puede ser ajena a estos cambios. Habrá que tenerlos en consideración para tender a encajar los sistemas de actividad que entran en juego con el objetivo de cumplir las finalidades que el sistema educativo se propone. Entiendo, pues, la innovación no como el cambio por el cambio, sino, bien al contrario, como resultado de la necesidad de ajustar progresivamente la enseñanza a las condiciones cambiantes de los sistemas de actividad implicados. Un análisis histórico de las transformaciones de la enseñanza o de las grandes propuestas de cambio formuladas por los pedagogos, nos mostraría que estas propuestas responden a la conciencia de que las sociedades plantean exigencias nuevas que la educación no puede descuidar. Pensemos, por ejemplo, en las propuestas pedagógicas de Comenius, quien plantea una educación para todos y todas, lo cual tie- ne como consecuencia la necesidad de que haya nuevas formas de enseñar lejos de la que era propia de una relación individual del alumno con el profesor.

La reflexión hecha hasta el momento nos sitúa en el aula donde el profesor, cuya tarea es enseñar lengua a los niños y niñas y a adolescentes, tiene que tomar decisiones sobre qué y cómo enseñar. El profesor llega al aula con unos conocimientos teórico-prácticos fruto de sus experiencias como alumno, de su formación y de su práctica como profesor. Como hemos dicho, la dinámica de los sistemas implicados en el sistema didáctico le obliga a reajustar su práctica, a reflexionar sobre la adecuación de sus propuestas, en definitiva, a introducir cambios en su manera de enseñar y a repensar sus contenidos. Hemos reducido el ejemplo al profesor, pero lo podemos hacer extensible a toda la "noosfera", usando el término de Chevallard (1989).

Dice Márkus: "solo es posible que haya una actividad productiva específicamente humana cuando es posible la contraposición y la comparación del objetivo en cuanto que imagen ideal de la forma deseada del objeto con la cosa objetiva actualmente presente, percibida cuando la actividad deviende actividad dirigida a un fin y controlada por este fin".

Nos situamos, así, en el terreno de la reflexión en la acción y entramos ya en un campo en que podemos empezar a hablar de la investigación, al considerar la posibilidad de comparación entre el objeto sobre el cual se trabaja y el objetivo al cual se tiende.

\section{El conocimiento científico en el campo de las didácticas}

Antes de entrar a hablar de la investigación en didáctica, nos hacen falta dos reflexiones básicas: una referida a la relación entre el conocimiento sistemático, sistematizado, que llamamos científico; y la segunda, referida a un problema que es común 
a muchas de las ciencias sociales: la posibilidad de sistematizar el conocimiento sobre aquello que es dinámico y cambiante.

- En relación con el primero, nos basamos en la distinción vygotskiana entre conceptos espontáneos y conceptos científicos, enriquecida por la conceptualización de Cassirer sobre el lenguaje y la ciencia como representaciones generalizadas de la realidad a diferentes niveles. A riesgo de hacer una gran simplificación, obligada por el espacio de esta intervención, podemos decir que el trabajo en común, la actividad humana mediada por el lenguaje en su función comunicativa, está en el origen de la función representativa del lenguaje, de su capacidad de referirse a la realidad y no simplemente de designarla. La palabra no es una etiqueta que señale los objetos, sino que es una generalización que configura de alguna manera la realidad para conocerla y para dominarla. La conceptualización es, pues, inherente al quehacer humano, que es fundamentalmente un quehacer discursivo. En el devenir filogenético, la humanidad ha elaborado sistemas conceptuales para ordenar y entender esta realidad, sistemas que han tomado como referente no solo la realidad natural, sino también las mismas construcciones sociodiscursivas humanas mediadas por el lenguaje y ha elaborado modelos que las explican de manera sistemática y que las orientan. Se dibujan, así, dos tipos diferentes de conceptualización: los conceptos surgidos espontáneamente de la práctica, inherentes al uso del lenguaje, y los conceptos científicos surgidos de la actividad voluntaria de conocimiento de esta realidad. De todas maneras, a pesar de que, como señala Vygotski (1978), los dos tipos de conocimiento tienen, en la ontogénesis, orígenes diferentes, no se puede establecer solución de continuidad entre uno y otro y se tienen que considerar los dos como procesos dinámicos en que el ser humano trata de dar sentido al mundo en diálogo constante con la realidad. Podemos pensar el conocimiento en y sobre la práctica de enseñar y aprender desde esta distinción. Por ejemplo: puede ser que el saber espontáneo no ayude a concebir la distancia que hay entre un saber declarativo (definir qué es el sujeto de una oración) y un saber procedimental (identificar el sujeto); la investigación sobre el tema la hace evidente (Notàrio, 2001). El conocimiento científico, surgido de la investigación sistemática, ayuda a mirar de manera diferente lo que es saber gramática.

- La segunda reflexión parte de dos preguntas cruciales: si los procesos son cambiantes, si la realidad es dinámica, ¿cómo se puede elaborar conocimiento sistematizado, que requiere extraer datos de la realidad que necesariamente se referirán a un momento de los procesos implicados en la dinámica de la actividad y que requieren un tiempo propio de análisis?, ¿cómo podemos dar cuenta de ellos de manera sistemática, de forma que los resultados puedan ser compartidos públicamente para tomarlos como referencia para interpretar otras prácticas que serán ya diferentes? No pretendemos tener una respuesta a esta pregunta que requiere modelos teórico-metodológicos que permitan dar cuenta, interpretar, la complejidad de la actividad humana. Ricoeur (1986) aborda este problema haciendo un paralelismo entre la investigación en el campo de las ciencias sociales y el estudio hermenéutico del texto. La lectura de un texto es una actividad de comunicación, el lector en parte hace transparentes los elementos formales del texto para comprender su significado, está inmerso en el circuito de la comunicación. ¿En que consiste la investigación? El investigador extrae el texto del circuito comunicativo, lo hace opaco para analizar las formas, para entender los meca- 
nismos por los cuales comunica; la finalidad es devolverlo al circuito de la comunicación, pero ahora enriquecido por una mayor profundidad comprensiva. De este modo, aborda la superación de la dicotomía que se establece a menudo entre explicar (basado en la busca de causas) y comprender (basado en la participación empática en la misma actividad) proponiendo un nivel de comprensión que se base en el proceso intermedio de interpretación. La acción es significativa y puede ser objetivada en un proceso de suspensión de la comprensión. Las palabras de Ricoeur explican este proceso:

Laction sensée devient objet de sciences seulement sous la condition d'une sorte d'objectivation équivalente á la fixation du discours par l'éscriture.

[...] Ma thése est que l'action elle-même, l'action sensée, peut devenir objet de science sans perdre son caractère de signifiance à la faveur d'une sorte d'objectivation semblable à l'objectivation opérée par l'écriture. Grace à cette objectivation, l'action n'est plus une trasaction à l'aquelle le discours de l'action continuerair d'appartenir. Elle constitue un configuration que demande à être interpretée en fonction de ses connexions internes. (p. 213)

Aplicando lo que Ricoeur dice sobre la investigación al campo de las didácticas, podemos decir que los actores del sistema didáctico están inmersos en el fluir de la actividad, utilizan las lenguas para interactuar, para resolver problemas sobre el aprendizaje de contenidos específicos, por ejemplo, las mismas lenguas etc.; la investigación necesita detener de alguna manera este fluir de la actividad para analizarla y profundizar en su conocimiento con el objetivo de que este conocimiento retorne a ella enriqueciéndola. Desde este planteamiento, no podemos pensar que la práctica por si sola aporta conocimiento teórico. Necesitamos la distancia y la sistematización del conocimiento. En las palabras de Widdowson (1997, trad.cast.: 1998), encontramos la misma idea:
Todas las actividades que se realizan al amparo de la palabra investigación pretenden ir más allá de las apariencias, pretenden descubrir categorías abstractas y conexiones subyacentes a fenómenos más o menos corrientes [...]. Podemos considerar la investigación como un proceso dialéctico permanente: formula la realidad en términos abstractos $y$, a su vez, de esta formulación abstracta se deriva una reformulación de la realidad.

\section{Los niveles de la investigación en y sobre la acción}

Estamos ahora, pues, en el terreno de la investigación y aceptamos que requiere distancia respecto de la práctica, pero esta distancia puede ser mayor o menor y puede establecerse con objetivos algo diferentes, aunque siempre tendrá como finalidad fundamental elaborar algún tipo de conocimiento.

Mialaret (1992) reconocía dos significados diferentes en el término “investigación”: por un lado, la búsqueda de soluciones para cualquier situación problemática, inherente a la actividad humana, a través de la cual, evidentemente, se aprende, y, por otra, la actividad sistemática que recurre a metodologías establecidas, a fin de elaborar conocimiento sistemático, comunicable y, a su vez, revisable. Es en este último sentido cuando habla de investigación científica.

Entre los dos extremos del significado de este término, diversos autores han situado diferentes conceptos asociados a la investigación sobre la actividad y algunos sobre la actividad educativa, en relación con la proximidad inmediata mayor o menor con la práctica. Schön habla de la reflexión en la acción que considera constituida por el ciclo acción, observación, análisis, evaluación, planificación, que se formula a menudo en términos de práctica reflexiva.

Otros autores, sin embargo, profundizan un poco más en las posibilidades de la investigación sobre 
la práctica y establecen niveles diferentes. Dewey (citado en Griffiths y Tann, 1992) reconocía tres: reflexión durante la acción, el segundo la reflexión después de la acción y un tercer nivel que los combina y utiliza teorías públicas. Griffiths y Tann (1992) retoman esta distinción y la amplían distinguiendo cinco niveles agrupados en dos bloques: uno referido a la investigación en la acción y la otra, a la investigación sobre la acción.

En el primer bloque, el de la reflexión en la acción, circunscrito en general al ámbito personal del desarrollo de las actividades, se sitúa la reflexión estrechamente unida a la práctica inmediata en dos niveles:

1. El de la actuación-reacción, que consistiría en la respuesta inmediata ante una situación problemática. Por ejemplo: si un alumno pregunta cómo se escribe una palabra, el profesor puede responder dándole la solución, remitiéndolo al diccionario o haciéndolo reflexionar sobre el contexto, etc. Entre la situación planteada y la respuesta no hay casi distancia, aunque, evidentemente, la reacción del profesor dependerá de sus ideas, creencias, los conceptos que tenga sobre el aprendizaje, sobre la autonomía del alumno, etc.

2. El segundo nivel, formado por el ciclo actuación, control y reacción, consiste en readaptar los planes para actuar. Supone un cambio en los planes previstos, por ejemplo: si el alumnado ha mostrado un interés especial por algún aspecto del trabajo, el profesor puede decidir sobre la marcha proseguir por ese camino en lugar de continuar la actividad tal como la tenía prevista.

El segundo bloque, el de la reflexión sobre la acción, es, en general, interpersonal y colectivo y se puede dar a tres niveles:
3. El tercer nivel consiste en un ciclo de actuación, observación, análisis y evaluación, seguidos de planificación y nueva actuación; implica reflexión y distancia respecto del momento de la práctica y puede llevar a modificar los planes de enseñanza y no a una simple modificación en el mismo proceso de realización.

4. El cuarto nivel, compuesto por actuación, observación sistemática, análisis riguroso, evaluación, planificación y nueva actuación, implica que la observación se focaliza y se lleva a cabo de manera sistemática a través de un proceso de recogida de datos mediante fichas, grabaciones en audio o vídeo, diarios de clase, etc. El análisis y la observación pueden ocupar semanas y meses. En este nivel, interesa ya la validez y la fiabilidad de la investigación.

5. El ciclo actuación, observación sistemática, análisis riguroso, evaluación, reconceptualización, planificación y actuación implica un proceso de cambio conceptual y, por tanto, revisión de las teorías previas. El profesor o el investigador van más allá del análisis sobre cómo funcionan unas prácticas determinadas. A través de una lectura crítica de las teorías subyacentes a la actuación, se ven implicados en un proceso de revisión conceptual.

Los dos últimos niveles conducen a elaborar conocimiento científico en el sentido comentado anteriormente.

De acuerdo con lo que hemos ido apuntando, la investigación en didáctica no puede ser solo descriptiva, sino que intenta comprender e interpretar una actividad humana compleja, como es la de enseñar y aprender lenguas con el fin de devolver el conocimiento generado para que infunda de nuevo la práctica a un nivel mayor de profundidad. 
Desde este punto de vista, tampoco tiene suficiente fundamento la distinción que hacen algunos autores (Daunay y Reuter, 2008) entre implicación militante y propósito científico. El objeto de la investigación en didácticas son las prácticas de enseñanza y aprendizaje de los contenidos propios de cada área de conocimiento, con el objetivo de hacerlas más adecuadas al objetivo que se plantea la enseñanza en la escuela. Incluso en los niveles más altos de control y de rigor, las finalidades de conocer, interpretar y mejorar la práctica son inherentes a la existencia misma de la investigación didáctica.

\section{Conclusión}

Hasta ahora hemos ido poniendo los hitos que nos permitirán explicitar de manera sucinta la relación entre intervención, innovación e investigación. Si entendemos que enseñar es una actividad humana orientada a un objetivo, a una finalidad, deberemos aceptar que la intervención del profesor es un elemento clave del sistema didáctico. El profesor tiene planteados unos objetivos que, de manera más o menos explícita en cada momento, guían su actuación. El sistema es dinámico, cambiante en el tiempo, y dependiente de los contextos, lo cual exige del docente la capacidad de ajustar su intervención a estas situaciones cambiantes. El profesorado interpreta estas situaciones a partir de sus creencias, representaciones, teorías más o menos estables, a partir de las cuales actúa y hace planteamientos nuevos para responder a ellas. En este sentido, la innovación es inherente a la misma dinámica del sistema. Pero, ¿cómo podemos saber que un cambio es más adecuado a la nueva situación? Aquí es donde la investigación ocupa su lugar.

Como hemos visto, la investigación se puede desarrollar en niveles diferentes de control y conciencia. Pero, por la complejidad de los factores que inciden en el sistema didáctico y para avanzar realmente en un conocimiento sistemático relacionado con la didáctica de la lengua (o de las diferentes didácticas), será necesario que, en la práctica reflexiva (que podríamos asimilar a los niveles 2 y 3 explicados anteriormente), se añada un conocimiento elaborado sistemáticamente, que pueda dar elementos para enriquecerla $y$, por tanto, ayude a transformar las prácticas. Un tema que ahora no trataré es el de los actores de este tipo de investigación. El profesorado debe tener medios para poder participar en ella y no solo ser receptor de los resultados elaborados por otras personas.

\section{Referencias}

Bronckart, J. P. y Schneuwly, B. (1985). Les didáctiques entre la ciéncia i la utopia: El cas de la didáctica del francès com a llengua materna. Temps d'Educació, 5, 185-205.

Camps, A. y Uribe, P. (2008). La construcción del discurso escrito en un entorno académico: una visión de la dinámica del aprendizaje de los géneros discursivos. En L. Barrio (Coord.). El proceso de enseñar lenguas. Investigaciones en didáctica de la lengua (pp. 27-56). Madrid: La Muralla.

Cassirer, E. (1944). Essay of Man. New Haven: Yale University Press.

Chevallard, Y. (1989). La transposition didactique: Du savoir savant au savoir enseigné. París: La pensé sauvage, 1991.

Daunay, B. y Reuter, Y. (2008). "La didactique du français: questions d'enjeux et de méthodes". Practiques, 137-138, 57-78.

Engeström, Y. (1987). Learning by Expanding: An Activity-Theoretical Approach to Developmental Research. Helsinki: Orienta-Konsultit. 
Griffiths, M. and Tann, S. (1992). Using reflective practice to link personal and public theories. Journal of Education for Teaching, 18 (1), 69-83.

Leontiev, A. (1972 [1975]). Le développement du psychisme. París: Éditions Sociales.

Leontiev, A. (1981). Problemas del desarrollo del psiquismo 2. La Habana (Cuba): Editorial Pueblo y Educacion

Márkus, G. (1971). Marxizmus és "antropología". Budapest: Akadémia Kiadó.

Márkus, G. (1973). Marxismo y "antropología" (M. Sacristán, Trad.). Barcelona: Grijalbo.

Mialaret, G. (1992). Place et role de la recherche en education dans la formations des enseignants. European Journal of Teacher Education, 15 (12), 33-43.

Notario, G. (2001). Los conceptos gramaticales de los alumnos de secundaria: el sujeto. En A. Camps (Coord.). El aula como especio de investigación y reflexión: Investigaciones en didáctica de la lengua (pp. 181-193). Barcelona: Graó.

Ribas, T. (2001). La regulación del proceso de composición escrita en grupo: análisis de la utili- zación de las pautas de revisión. En A. Camps (Coord.). El aula como espacio de investigación y reflexión (pp. 51-67). Barcelona: Graó.

Ricoeur, P. (1986). Du texte à l'action: Essais d’herméneutique II. París: Éditions du Seuil.

Ruiz Bikandi, U. (2007). La autoobservación en clase de lengua como mecanismo colectivo de formación: El discurso protector. Cultura y Educación, 19 (2), 165-182.

Schön, D. A. (1987). Educating the Reflective Practitiones. San Francisco / Londres: Jossey-Bass Publishers.

Schön, D. A. (1992) La formación de los profesionales reflexivos. Barcelona: Paidós.

Vallés, J., Álvarez, D. y Rickenmann, R. (2011). Lactivitat docent, intervenció, innovació, investigación. Girona: Documenta Universitària.

Vygotski, L. V. (1977). Pensamiento y lenguaje. Buenos Aires: La Pléyade.

Widdowson, H. G. (1998). Aspectos de la enseñanza de la lengua. En F. Cantero, A, Mendoza y C. Romea (Comp.). Didáctica de la lengua y la literatura en una sociedad plurilingüe del siglo XXI (pp. 1-22). Barcelona: Universitat de Barcelona. 